\title{
Einstellungen zur familialen Solidarität im Alter: Eine vergleichende Analyse mit türkischen Migranten und Deutschen
}

\author{
Attitudes toward family solidarity in old age: A comparative analysis of \\ Turkish migrants and Germans
}

\begin{abstract}
Zusammenfassung:
Die Studie untersucht das familiale Solidaritätspotenzial für pflegebedürftige Eltern bei türkischen Migranten der ersten und zweiten Generation und kontrastiert es mit jenem der deutschen einheimischen Bevölkerung. Die zentralen Fragen lauten, welche Rolle ethnisch-kulturellen bzw. sozialstrukturellen Einflussgrößen zukommt und ob sich die Muster über verschiedene Altersgruppen hinweg verändern. Den theoretischen Hintergrund bilden Diskussionen um Transmissions- und Akkulturationsprozesse in der Migration. Auf Grundlage der Daten des Generations and Gender Survey 2005 und 2006, der die 18 bis 79-jährige Wohnbevölkerung in Privathaushalten Deutschlands sowie in einer Zusatzerhebung ergänzend die türkischen Staatsangehörigen berücksichtigt, kann gezeigt werden, dass das familiale Solidaritätspotenzial bei türkischen Migranten wesentlich stärker ausgeprägt ist als bei Deutschen. Die Unterschiede bleiben in der nachfolgenden Generation und über alle Altersgruppen hinweg bestehen. Sozialstrukturelle Merkmale sind von geringer Bedeutung. Die Befunde zeigen, wie stark die Transmissionsprozesse zwischen den Generationen sind: Es gibt wenig Hinweise auf einen intergenerationalen ,acculturation gap“".
\end{abstract}

Schlagwörter: Generationenbeziehungen, türkische Migranten, Deutschland, Pflege, Werte und Normen

\begin{abstract}
:
The study examines the attitudes toward family solidarity and filial care obligations among Turks of the first and second immigrant generation as compared to Germans. The foci lie on the impact of ethnic-cultural and socio-structural predictors, respectively, and whether patterns change across different age groups. Processes of intergenerational transmission and acculturation in migration constitute the theoretical background. Data from the Generations and Gender Survey 2005 and 2006 are used, including respondents in private households in Germany aged 18 to 79 years of the main sample, and the migrant sample, conducted on same-aged Turkish citizens in Germany. It was found that the family solidarity potential is far higher among Turkish migrants than among Germans. These differences persist in the second generation and in all age groups. Socio-structural predictors are of little relevance. The analyses indicate strong transmission processes between family generations: There ist little evidence of an "acculturation gap".
\end{abstract}

Key words: intergenerational relationships, Turkish migrants, Germany, elderly care, filial norms and values 


\section{Einleitung}

Mit einem steigenden Anteil älterer Menschen gleicht sich die Altersstruktur der ausländischen Bevölkerung Deutschlands seit einigen Jahren an die der deutschen Bevölkerung an (Statistisches Bundesamt 2011, BMFSFJ 2000). Altern in Deutschland bedeutet für Menschen mit Migrationshintergrund eine Kumulation von migrations-, schicht- und altersspezifischen Merkmalen. So zeigt sich im Alter für viele eine vergleichsweise schwierige Lebenslage, oftmals einhergehend mit einem schlechteren Gesundheitszustand als Folge der körperlich stark belastenden beruflichen Tätigkeiten oder schlechter Wohnbedingungen (BMFSFJ 2005, Baykara-Krumme/Hoff 2006, Robert-Koch-Institut 2008, Zeman 2009, TucciYildiz 2012). Angesichts der inzwischen vielfach konstatierten Bleibeabsicht der Arbeitsmigranten im Alter stellt sich auch für Familien von Migranten zunehmend die Frage, wer die Unterstützung und Pflege der Älteren übernehmen soll bzw. kann.

Die Diskussion um die Folgen der Migration und eines Wandels von Generationenbeziehungen in Migrantenfamilien wird von widersprüchlichen Annahmen geleitet. Zum einen wird davon ausgegangen, dass Pflege innerhalb der Migrantenpopulation als innerfamiliäre Angelegenheit gesehen wird. Zum anderen gibt es die Annahme, dass starke intergenerationale (Kultur-)Konflikte existieren, die eine innerfamilial organisierte Pflege erschweren. Vor allem die Einstellungen zur Pflege in den verschiedenen Familien- und Einwanderergenerationen und die Einflussfaktoren, die sie bedingen, sind noch unzureichend untersucht (für die Niederlande siehe z.B. de Valk/Schans 2008; Arends-Tóth/ Van de Vijver 2008). Dies ist oft auf fehlende Daten zu Einstellungen und zum faktischen Pflegeverhalten (Baykara-Krumme 2007; Okken et al. 2008; Kohls 2012; Zimmermann 2012) bzw. auf eine Datenlage zurückzuführen, die lediglich deskriptive Beschreibungen zulässt (z.B. Vogel 2012). Einstellungen sind insofern von großer Bedeutung, als dass sie gerade in Bezug auf die Thematik der Pflege im Alter Rückschlüsse auf das vorhandene Solidaritätspotenzial zulassen. So lässt sich auf dieser Basis ein möglicher Wandel im Rahmen von Akkulturationsprozessen ebenso nachzeichnen wie altersbezogene Unterschiede. Um in dieser Frage weiterführende Erkenntnisse zu gewinnen, erfolgt in diesem Beitrag eine differenzierte Untersuchung der Einstellungen von Angehörigen der türkischen ersten und zweiten Einwanderergeneration in Deutschland im Vergleich zur einheimischen deutschen Bevölkerung. Auf Basis der ersten Welle des Generations and Gender Survey (GGS) wird untersucht, welche ethnisch-kulturellen und sozio-demographischen Faktoren das Zustimmungsverhalten hinsichtlich der Frage beeinflussen, ob Hilfeleistungen und Pflegeaufgaben an Eltern durch die Kinder übernommen werden sollen. Von besonderem Interesse ist dabei, wie sich die Einstellungen über die Einwanderergenerationen und nach Alter verändern, um zu prüfen, welche Bedeutung den Generationenbeziehungen in den jeweiligen Lebensphasen zukommt.

Zunächst werden verschiedene theoretische Ansätze und empirische Befunde zur Erklärung des Wandels der Generationenbeziehungen im Rahmen einer Migration dargestellt sowie anschließend Arbeitshypothesen formuliert. Im dritten Abschnitt erfolgt eine kurze Beschreibung des Datensatzes und der Methodik als Grundlage für die daran anschließende deskriptive und multivariate Analyse. Abschließend werden die zentralen Befunde diskutiert. 


\section{Untersuchungsansätze und Forschungsstand}

Einstellungen zur Generationensolidarität reflektieren kulturelle Normen darüber, welche Verpflichtungen und Erwartungen hinsichtlich gegenseitiger Unterstützungsleistungen zwischen den Familienmitgliedern bestehen (Cicirelli 1990; Rossi/Rossi 1990; Burr/ Mutchler 1999). Individuen entwickeln diese Einstellungen im Rahmen des Sozialisationsprozesses durch persönliche Erfahrungen, Beobachtungen und Wertevermittlung in ihrem jeweiligen kulturellen Kontext. In der Literatur finden sich verschiedene Erklärungsansätze und unterschiedliche Differenzierungen von „Familienkulturen“ (Markus/ Kitayama 1991; Inglehart/Baker 2000; Kağıtçıbaş1 1996, 2006; Reher 1998; Nauck/ Suckow 2006). Der prominenteste Ansatz aus der kulturvergleichenden Forschung ist das Individualismus-Kollektivismus-Paradigma, das von unterschiedlich geregelten Beziehungen zwischen dem Individuum und der Gruppe ausgeht. So wird beispielsweise argumentiert, dass in stärker kollektivistischen Gesellschaften, zu denen die Türkei - vor allem in ihren ländlichen Gebieten - zählt, das Wohlergehen der Gruppe, insbesondere der Verwandtschaftsgruppe, eine zentrale Rolle spielt, während in stärker individualistischen Gesellschaften, wie den Ländern Westeuropas, persönliche Ziele und Interessen im Vordergrund stehen. Innerhalb einer kollektivistischen „culture of relatedness“ sei demnach eher von vielfältigen und intensiven Formen intergenerationaler Solidarität auszugehen (großes Attachment, Loyalität, Verpflichtungen, Verantwortung unter den Familienmitgliedern) als in einer individualistischen „culture of separateness“ (Kağıtçıbaşı 1996). Die türkeistämmigen Migranten der ersten Generation wuchsen in diesem familiär-orientierten Umfeld auf, in dem Hilfen der (Schwieger-)Kinder an ihre bedürftigen Eltern durch instrumentelle Unterstützung und finanzielle Absicherung selbstverständlich waren auch angesichts geringer wohlfahrtsstaatlicher Alternativen. Der deutsche Wohlfahrtsstaat mit seinen stark ausgebauten sozialen Sicherungssystemen deckt dagegen verschiedene Risikolagen, unter anderem im Alter, ab. Strukturelle Hilfen für Ältere wie finanzielle Transfers und Pflege können leichter außerfamiliär organisiert werden, auch wenn die emotionalen Beziehungen zwischen den Generationen und selbst die gegenseitigen (ergänzenden) Unterstützungsleistungen stark ausgeprägt bleiben (Szydlik 2000).

Internationale Wanderungen gehen mit einem Wechsel von Kontexten einher, vor allem bei einer Migration aus der ländlichen Türkei in die urbanen Regionen Deutschlands, mit anderen sozioökonomischen Bedingungen, Bildungs- und Einkommensopportunitäten und kulturellen Mustern. Zugleich kann eine Migration auch ein kritisches Lebensereignis für das Individuum und seine Familie darstellen, etwa aufgrund der Trennung von Familienmitgliedern und sozialen Netzwerken sowie den spezifischen Orientierungs- und Integrationsanforderungen, dem Minderheitenstatus und Ausgrenzungserfahrungen in der eigenen und nachfolgenden Generationen. In der Forschungsliteratur finden sich verschiedene Annahmen über die Auswirkungen dieser Erfahrungen auf die Einstellungen zur Generationensolidarität, die als konträre Argumentationsstränge konzeptualisiert werden können (Baykara-Krumme et al. 2011).

Das Konzept der „intergenerationalen Transmission“ postuliert aufgrund intensiver Austausch- und Wertvermittlungsprozesse zwischen den familialen Generationen geringe Veränderungen in der ersten und nachfolgenden Einwanderergenerationen. So wird angenommen, dass sich Migranten und ihre Nachkommen vor allem im privat-familiären Be- 
reich stark an den kulturellen Traditionen ihres Herkunftslandes orientieren. Studien zeigen, dass z.B. türkischstämmige Eltern auch nach der Migration noch eine vergleichsweise ausgeprägte Nützlichkeitserwartung an Kinder aufweisen (BMFSFJ 2000). Die Eltern-Kind-Beziehung ist zugleich durch eine ausgeprägte Reziprozität gekennzeichnet: In der Erziehungsphase sind die Eltern für die Kinder verantwortlich, die Kinder übernehmen dann, so die Erwartung, im Alter die materielle und immaterielle Versorgung der und Fürsorge für die Eltern (Nauck 2000). In der Migration, d.h. im Aufnahmeland kann zudem eine verstärkte Akzentuierung der Werte und Normen der Herkunftskultur stattfinden. So erfolgt in der Migration die Vermittlung der Herkunftskultur oft nur im Rahmen intergenerationaler Transmissionsprozesse. Eltern geben ihre Werte an die Kinder dadurch vergleichsweise intensiv weiter, da nur so ihr Aufrechterhalten gewährleistet werden kann. Das Resultat ist jedoch keine perfekte Abbildung der Herkunftskultur: Vielmehr bewegt sich dieses Abbild im Spannungsverhältnis zwischen exakter und fehlender kultureller Transmission (Nauck 2002) und beinhaltet zugleich Momente einer Immigrantenkultur (Portes/Rumbaut 2001; Kofman 2004). Studien konnten zeigen, dass bei Migrantenfamilien im Vergleich zu nicht gewanderten Personen im Herkunftsland die Übereinstimmung und Konformität der Einstellungen zwischen den Generationenbeziehungen noch größer war (Nauck 2000; Idema/Phalet 2007).

Diesen Ansätzen stehen akkulturationstheoretische Erklärungen (Alba/Nee 1997; Berry 1997) und der Ansatz der Kulturkonfliktthese gegenüber (Park 1964; Schrader et al. 1979), wonach es im Zuge einer Migration zu ausgeprägten individuellen und intergenerationalen Veränderungen in den Werteinstellungen und im Verhalten kommt. Dabei wird angenommen, dass sich die zweite Generation (d.h. die im Zielland geborenen Kinder) über die Institutionen der Gesellschaft schneller akkulturiert als ihre Eltern. Dies führt nicht nur zu einem ,acculturation gap“, sondern auch zu entsprechenden innerfamilialen Konflikten (Zhou 1997; Portes/Rumbaut 2001), da die Einwanderer der ersten Generation aufgrund ihrer Sozialisation im Herkunftsland stärker an ihren dort erworbenen Einstellungen und Werten festhalten, während sich die zweite Einwanderergeneration eher mit jenen der Aufnahmegesellschaft identifiziert. Sowohl die Präferenzen als auch die Opportunitäten für die Generationensolidarität können sich verändern, z.B. durch eine zunehmende Erwerbsbeteiligung und Karriereinteressen von Frauen im Zuge von steigender Bildung: Das Pflichtgefühl in der Generationenbeziehung wandelt sich, sodass unter anderem der Pflege der Eltern nun nicht mehr in dem Maße nachgekommen wird bzw. werden kann wie im als traditionell angesehenen Modell des familialen Mehrgenerationenhaushalts (Aboderin 2004). Besonders die Kinder von Einwanderern befinden sich, so die Annahme, in einem Spannungsfeld: „Growing up in an immigrant family has always been difficult, as individuals are torn by conflicting social and cultural demands, while they face the challenge of entry into an unfamiliar and frequently hostile world" (Portes/Zhou 2005: 85). Diese intergenerationalen Divergenzen können sich im Alter noch verschärfen, wenn bei den alt gewordenen Einwanderern der ersten Generation als Folge einer verstärkten Rückbesinnung auf die Kindheit und kulturelle Herkunft eine Hinwendung zur eigenen ethnischen Gruppe erfolgt. Dieser Rückbezug geht tendenziell mit einer stärkeren Familienorientierung und entsprechenden Erwartungen an die Kinder einher (Dietzel-Papakyriakou 1993; Prahl/Schroeter 1996), selbst wenn viele Ältere die Belastungen der Kinder antizipieren und den Erhalt ihrer Selbständigkeit und einer gewissen Unabhängigkeit von der Familie suchen (Matthäi 2005). 
Die Forschungslage zu den Auswirkungen der Akkulturationserfahrungen für die Eltern-Kind-Beziehungen ist allerdings uneindeutig und hat zu der Annahme geführt, dass ,the hypothesised link between acculturation gaps and the quality of family relationships might be overstated" (Tardif-Williams/Fisher 2009: 151). Betont wird, dass auch die erste Generation intensive Akkulturationsprozesse erlebt und zugleich bei der zweiten Generation intensive Beziehungen zum Herkunftskontext bestehen bleiben, z.B. über eine transnationale Partnerwahl (Dietzel-Papakyriakou 1993; Nauck 2001). Zugleich befinden sich die Institution Familie und damit die Generationenbeziehungen insgesamt im Wandel, im Herkunfts- wie im Aufnahmeland (Nauck 2002). Nachdem die Diskussion zur Situation der Migrantenfamilien in Deutschland lange Zeit durch eine einseitige, problemzentrierte Betrachtung der Generationenbeziehung gekennzeichnet war, hat sich der Fokus inzwischen geweitet. Empirische Untersuchungen zeigen, dass intergenerative Entfremdung und Konflikte zwar vorkommen, aber keineswegs die häufigsten Muster darstellen (Nauck 2000; Olbermann 2003; Matthäi 2005; Baykara-Krumme 2007). Anhand des faktischen Unterstützungsverhaltens finden sich keine Hinweise, wonach die Neigung, den Eltern im Alter Hilfeleistungen zu gewähren, in der zweiten Generation sinken sollte. Vielmehr scheinen elterliche Erwartungen an die Generationenbeziehungen von den Kindern in einem hohen Maße antizipiert zu werden. Es kann daher von einer gewissen Bereitschaft ausgegangen werden, diesen Erwartungshaltungen nachzukommen (Steinbach 2004; Nauck 2000; BMFSFJ 2010).

In der Literatur wird inzwischen stark betont, dass sowohl kulturelle als auch strukturelle Bedingungen ausschlaggebend für Veränderungen im Zuge der Migration und Akkulturation sind, wenn auch vielfach noch unklar ist, welche Rolle sie jeweils spielen: „Researchers increasingly recognize the complex interplay of both structural and cultural factors in determining the unique family patterns and relationships observed among various immigrant groups" (Glick 2010: 506). Hinsichtlich der Generationenbeziehungen haben verschiedene Studien gezeigt, dass Unterschiede zwischen der einheimischen und der zugewanderten Bevölkerung zum Teil auf soziodemographische und sozioökonomische Unterschiede zurückzuführen sind (z.B. Lye 1996; Glick/Van Hook 2002; BaykaraKrumme 2007).

Weiterhin zeigt sich in internationalen Studien aus den USA, Israel, den Niederlanden und Großbritannien, dass die Erwartungshaltungen an Pflichten der Kinder zwischen den ethnischen Einwanderungsgruppen mit dem Akkulturations- und Bildungsniveau variieren (Katz 2009; Laidlaw et al. 2010; de Valk/Schans 2008; Liefbroer/Mulder 2006; Angel 1996). Eine Untersuchung unter lateinamerikanischen Einwanderern aus den USA verweist auf die größere Erwartungshaltung an die Kinder mit a) zunehmendem Alter, b) unter Frauen und c) unter sprachlich weniger akkulturierten Befragten (Kao/Travis 2005). De Valk und Schans (2008) wiesen eine besonders starke Familienorientierung bei türkischstämmigen Migranten in den Niederlanden nach, sowohl im Vergleich zur einheimischen niederländischen Bevölkerung als auch im Vergleich zu anderen Einwanderern, mit einer höheren Erwartung an Kontakthäufigkeit und -pflege der Älteren durch die Kinder. Zwar nahm die Erwartungshaltung an die Kinder mit zunehmendem Bildungsniveau ab. Allerdings konnten diese Daten unter anderem für die türkeistämmigen Migranten einen deutlichen „ethnischen Faktor“ ausmachen (de Valk/Schans 2008), der auch in den Folgegenerationen noch bestehen bleibt (Arends-Tóth/Van de Vijver 2008): 
Die zweite Generation unterscheidet sich demnach in den Einstellungen, aber nicht mehr im generationenbezogenen Verhalten von den einheimischen Niederländern.

\section{Ableitung der Hypothesen}

Ausgehend von den vorgenommenen Überlegungen werden drei zentrale Hypothesen hinsichtlich der Einstellungsunterschiede zur Erbringung von Unterstützungsleistungen formuliert. Vor dem Hintergrund einer starken kulturellen Prägung durch den Herkunftskontext und die unterschiedlichen „Familienkulturen“ in der Türkei und Deutschland (Kağıtçıbaşı 1996; Nauck/Suckow 2006) postulieren wir, dass türkische Migranten der ersten Generation stärkere Erwartungen an die Solidarität durch die Kinder äußern als einheimische Deutsche (H1). Neben den kulturellen Einflüssen kann dies auch das Ergebnis struktureller Rahmenbedingungen sein: Angesichts der schlechteren materiellen Situation vieler Migranten ist der Austausch von Unterstützung und Transfers unter den Familienmitgliedern von größerer Bedeutung als bei der deutschen Bevölkerung (BMFSJ 2000; speziell zu Älteren: Tucci/ Yildiz 2012). Angesichts von Akkulturationsprozessen, die in der zweiten Generation intensiver sind als in der ersten (Alba/Nee 1997; Portes/Rumbaut 2001), erwarten wir geringere Einstellungsunterschiede zwischen türkeistämmigen Angehörigen der zweiten Einwanderergeneration im Vergleich zur deutschen einheimischen Bevölkerung. Wir gehen allerdings davon aus, dass gleichzeitig stattfindende Transmissionsprozesse (Nauck 2000; Phalet/ Schönpflug 2001) dazu führen, dass eine gewisse Übereinstimmung zwischen der ersten und der zweiten Generation besteht, die Unterschiede zu Deutschen also geringer sind, aber nicht völlig verschwinden (H2). Im Hinblick auf die Rolle des Alters erwarten wir eine Intensivierung der Erwartungen an die Generationensolidarität mit zunehmendem Alter aufgrund einer stärkeren Rückbesinnung auf die kulturellen Familientraditionen im Herkunftsland bei den türkeistämmigen Migranten (Dietzel-Papakyriakou 1993; Prahl/Schroeter 1996). In dessen Folge nehmen die Unterschiede zu Deutschen nicht ab (Konvergenz), sondern vergrößern sich sogar möglicherweise noch (H3a). Angehörige der mittleren Altersgruppen bzw. Geburtskohorten begreifen sich aufgrund ihrer Position als Kinder, die selbst bereits potenzielle und baldige Hilfegeber sein werden. Vor dem Hintergrund der Wertetransmission gehen wir von deutlichen Unterschieden zwischen Deutschen und Türkeistämmigen in der Unterstützungsbereitschaft aus (H3b). In den jüngeren Geburtsjahrgängen wird das Thema der Pflege der Eltern noch wenig antizipiert, allerdings führt die kulturelle Transmission dazu, dass für türkeistämmige junge Erwachsene dieses Thema bereits von größerer Bedeutung und wichtiger ist als für einheimische Gleichaltrige, mit entsprechenden Unterschieden in den Werteinstellungen (H3c). Eine Trennung von Alters- und Kohorteneffekten ist dabei mit den vorliegenden Querschnittsdaten nicht möglich.

Das Bildungsniveau, der Familienstand, eigene Kinder sowie der eigene Gesundheitszustand, die Teilnahme an religiösen Veranstaltungen und Einsamkeitsgefühle werden als zentrale Kontrollvariablen in die Modelle aufgenommen. Sie beeinflussen ihrerseits die Werteinstellungen zur Generationensolidarität (Rossi/Rossi 1990; de Valk/Schans 2008; Burr/Mutchler 1999). Mit steigender Bildung, so die Hypothese, steigen die Wahrnehmung und die Möglichkeiten (instrumenteller und monetärer) außer-familiärer Unterstützungsmöglichkeiten, außerdem sind die Opportunitätskosten für die Versorgung der El- 
tern (z.B. bei der möglicherweise notwendigen Aufgabe des Berufs) größer (de Valk/ Schans 2008). Existiert ein Partner, so sollten die Hilfeerwartungen an Kinder geringer sein, da der Partner wichtige Aufgaben übernehmen kann (Olbermann 2003), während das Vorhandensein von Kindern die Erwartungen an diese erhöht. Ein schlechter Gesundheitszustand stellt einen zentralen Faktor für Hilfebedarf dar, der die Erwartungen an die Hilfe durch Kinder entsprechend positiv beeinflusst. Dies gilt sowohl für Ältere als auch für Jüngere, wobei letztere den Bedarf im Alter zunächst lediglich antizipieren. Vor dem Hintergrund der erhöhten Morbidität bei älteren türkischen Arbeitsmigranten (RobertKoch-Institut 2008) erwarten wir einen Effekt für die hier interessierenden Einstellungen zu Unterstützungserwartungen durch Kinder. Menschen, die mit großer Regelmäßigkeit an religiösen Veranstaltungen teilnehmen, verfügen einerseits über ein größeres, außerfamiliäres Netzwerk, das alternative Optionen bietet und die Erwartungen an die Kinder reduzieren kann. Andererseits ist davon auszugehen, dass die Religiosität mit einer stärker familial-konservativen Einstellung einhergeht, so dass die traditionalen Erwartungen an die Kinder besonders ausgeprägt sind. Im Hinblick auf Einsamkeitsgefühle kann angenommen werden, dass diese mit hohen Erwartungen an die Kinder einhergehen, und diese ggf. aus nicht erfüllten Erwartungen resultieren (Dykstra 2009; Jylhä/Saarenheimo 2010).

\section{Daten und Methode}

\section{Datenbasis}

Als Datenbasis dienen sowohl die erste Welle der deutschen Befragung des Generations and Gender Survey (GGS) als auch die im Jahr 2006 durchgeführte Zusatzerhebung unter türkischen Migranten in Deutschland. Der GGS ist Teil des international vergleichenden Generations and Gender Programme (GGP), welches von der Wirtschaftskommission der Vereinten Nationen für Europa (UNECE) in Genf koordiniert wird und in Deutschland im Auftrag des Bundesinstituts für Bevölkerungsforschung (BiB) durchgeführt wurde. Das vordergründige Ziel des GGS ist es, „,durch eine multidisziplinäre, retrospektive, prospektive und international vergleichende Studie aktuelle Daten zu Familienbeziehungen in Industrieländern zu gewinnen" (Ette et al. 2007: 7).

Die erste Welle der deutschen GGS-Hauptumfrage wurde 2005 durchgeführt und umfasst 10.017 deutschsprachige Personen aus Privathaushalten zwischen 18 und 79 Jahren. In dieser Befragung sind die türkischen Migranten stark unterrepräsentiert und für detaillierte Untersuchungen zu gering vertreten (Ette et al. 2007). Aus diesem Grund wurde eine Zusatzerhebung mit 4.000 in Privathaushalten in Deutschland lebenden türkischen Staatsangehörigen im Alter zwischen 18 und 79 Jahren durchgeführt. Die Fragen der Zusatzbefragung gleichen in Aufbau und Inhalt denen der Hauptbefragung des deutschen GGS. Ergänzend wurden migrations- und integrationsbezogene Fragen wie z.B. Sprachkenntnisse hinzugefügt. Um Kommunikationsprobleme und eine Selektivität nach Sprachkenntnissen zu verringern, wurde eine türkische schriftliche Übersetzungshilfe bereitgestellt. Bei den vorliegenden Daten handelt es sich um Querschnittsdaten. Individuelle Veränderungen über den Lebenslauf und in Folge einer Migration lassen sich nicht nach- 
zeichnen. Aufgrund der weiten Altersgrenzen ist aber ein Altersgruppenvergleich möglich. ${ }^{1}$ Außerdem lassen sich Angehörige der ersten und zweiten Einwanderergeneration differenzieren, also jene, die selbst zugewandert sind (1. Generation) und jene, die als Kinder von Zuwanderern in Deutschland geboren wurden (2. Generation). In die Analyse gehen sowohl die türkeistämmige Migrantenpopulation der Hauptbefragung als auch die türkischen Staatsangehörigen der Migrantenbefragung ein. ${ }^{2}$

\begin{abstract}
Abhängige Variable
Die vorliegende Analyse untersucht den Grad an Zustimmung zur Unterstützung und Pflege Älterer durch die Kinder anhand von drei verschiedenen Einstellungsvariablen, die anhand einer Fünf-Punkte-Likert-Skala von ,,(1) stimme voll und ganz zu“ bis ,,(5) stimme überhaupt nicht zu“ erhoben wurden. Die Fragen lauteten: „Kinder sollten die Verantwortung für ihre Eltern übernehmen, wenn diese Hilfe brauchen“, „Kinder sollten ihr Arbeitsleben umorganisieren, um den Bedürfnissen ihrer Eltern nachkommen zu können“ und „Kinder sollten ihre Eltern zu sich nehmen, wenn diese nicht mehr selbst für sich sorgen können“. Die Fragen wurden aus dem zur Verfügung stehenden Fragenkomplex „Werte und Meinungen“ des GGS gewählt, da sie inhaltlich am besten die hier interessierenden Einstellungen hinsichtlich zukünftiger Hilfeleistungen und einer Übernahme der Pflege der Eltern abbilden.
\end{abstract}

\title{
Unabhängige Variablen
}

Zentrale unabhängige Variable ist die Herkunft bzw. der Migrationsstatus. Als Vergleichsgruppe dienen die Deutschen, zu denen alle Personen zählen, die die deutsche Staatsangehörigkeit besitzen und deren beide Elternteile in Deutschland geboren sind. Die Gruppe der türkischen Migranten der ersten Generation wurde in der Türkei geboren und ist später nach Deutschland eingewandert. Die türkischen Migranten der zweiten Generation werden hier definiert als in Deutschland geborene und aufgewachsene Personen mit mindestens einem in der Türkei geborenen Elternteil.

Das Alter wird im multivariaten Gesamtmodell als metrische Variable berücksichtigt. Für die weiterführenden Analysen werden vier Altersgruppen gebildet, deren Altersgrenzen sich an den Altersstrukturen bzw. den zeitlich vergleichsweise früheren Familienbildungsprozessen in der türkischen Migrantenbevölkerung orientieren (Milewski 2009). Die Gruppe der bis 30-Jährigen umfasst Personen ohne oder mit eigenen kleinen Kindern, deren Eltern noch ,junge Alte" sind. Eine Pflegebedürftigkeit der Eltern ist in diesen Fäl-

1 Als eine der Schwächen des GGS sind inzwischen die biografischen Angaben zur individuellen Fertilität und der Partnerschaftsbildung in der Hauptbefragung des GGS erkannt worden, die in der hier vorliegenden Analyse jedoch nur die Variable des Partnerschaftsstatus betreffen. Bei der Partnerschaftsbildung und der Eheschließung im Lebenslauf zeigen sich als Schwächen vor allem unplausible, vom allgemeinen Forschungsstand abweichende Ergebnisse insbesondere bei älteren Kohorten (Naderi et al. 2009; Sauer et al. 2012; Kreyenfeld et al. 2010).

2 Der Einfachheit halber verwenden wir im Folgenden den Begriff „türkische Migranten“ und meinen damit alle türkeistämmigen Personen im Sample, unabhängig von ihrer Staatsangehörigkeit und ihrer ethnischen Zugehörigkeit. 
len noch die Ausnahme. Bei den 30- bis 44-Jährigen dagegen werden Versorgungsbedarfe der Eltern bereits stärker antizipiert und z.T. wird vermutlich bereits Unterstützung geleistet. Die 45- bis 59-Jährigen sind bereits stark eingebunden und antizipieren eigene Hilfebedarfe im Alter, während die über 60-Jährigen primär die eigene vorhandene oder zukünftige Pflegesituation im Blick haben. Türkische Migranten der zweiten Generation finden sich lediglich in den ersten beiden Altersgruppen. Das Geschlecht geht jeweils als dichotome Variable in die Analysen ein (Mann =1, Frau =0). Die Variable Bildung wurde anhand der im GGS abgefragten Items des höchsten Schulabschlusses und des höchsten Ausbildungsabschlusses generiert. Um diese beiden Variablen zusammenführen zu können, wurden die einzelnen Ausprägungen den Kategorien der International Standard Classification of Education (ISCED) zugeordnet (UNESCO 2012) und anschließend in die Ausprägungen ,niedrig“ (0) und „mittel/hoch“(1) gegliedert, v.a. aufgrund der geringen Fallzahlen der höher gebildeten türkischen Migranten.

Der Partnerschaftsstatus ist nicht explizit als Variable im GGS verfügbar (siehe Fußnote 1). Die Operationalisierung dieser Variable kann nur mittels der einzelnen Dimensionen der aktuellen Partnerschaft und der Partnerschaftsgeschichte generiert werden. Für die hier vorliegende Analyse wurde die Variable „Partnerschaftsstatus“ in die Ausprägungen „,keine Partnerschaft“ (0) und „verheiratet bzw. nichteheliche Partnerschaft" (1) untergliedert. Des Weiteren wurde eine dichotome Variable Kinder erstellt. Befragte, die keine Kinder haben (Ausprägung „1“), werden mit denen verglichen, die ein oder mehrere Kinder (Ausprägung „, $0^{\prime)}$ haben. Die Gesundheit der Befragten erschließt sich aus dem subjektiv eingeschätzten Gesundheitszustand auf einer Fünf-Punkte-Skala, von 1 ,sehr gut“ bis 5 „sehr schlecht“. Aufgrund der geringen Fallzahlen in den einzelnen Ausprägungen wurden die Fälle mit den Ausprägungen ,sehr gut" und „gut“ zu der Ausprägung ,gut“ (0) und jene mit den Ausprägungen ,teils teils“, ,schlecht“ und ,sehr schlecht“" in der neuen Ausprägung „eher schlecht" (1) zusammengefasst. Die Teilnahme an religiösen Veranstaltungen (Religiosität) wurde mit den Ausprägungen „mehrmals in der Woche“, „mehrmals im Monat“, „mehrmals im Jahr“, „,selten“ oder „,nie“ in den Analysen berücksichtigt. Als weiterer Komplex wird die Einsamkeitsvariable basierend auf dem Konzept der Loneliness Scale von De Jong-Gierveld und Kamphuis operationalisiert (De Jong-Gierveld/Kamphius 1985; De Jong-Gierveld/von Tilburg 2006). Der GGS enthält die sechs Items umfassende Kurzskala. Wiederum gilt es Einstellungsunterschiede zwischen den Gruppen unter Berücksichtigung der empfundenen Einsamkeit zu messen. Berücksichtigt wird eine dichotome Variable mit den Ausprägungen ,trifft zu“ und „trifft mehr oder weniger bzw. nicht zu“.

\section{Methodisches Vorgehen}

In Abschnitt 4.1 wird eine deskriptiv-vergleichende Auswertung der Zielvariablen und der Kontrollvariablen vorgenommen. Aufgrund der jeweils stark linksschiefen Verteilung werden die Zielvariablen dichotomisiert und anschließend in einem multivariaten binärlogistischen Regressionsverfahren dahingehend analysiert, welche sozialstrukturellen und wieteren Faktoren einen Einfluss auf die Einstellungen zur Hilfe und Pflege haben (Abschnitt 4.2). Besonders relevant ist dabei die Frage, ob die beobachteten Differenzen zwischen den Herkunfts-/Migrantengruppen mit diesen Merkmalen im Sinne von Kom- 
positions- bzw. Mediatoreffekten erklärt werden können. Anschließend werden Alterseffekte zusätzlich kontrolliert, indem für vier Altersgruppen untersucht wird, ob Differenzen nach Herkunft bzw. Migrationsstatus bestehen bleiben. Die logistischen Regressionsmodelle wurden mit Stata SE 11 geschätzt. Es werden Odds Ratios (OR) sowie die Standardfehler dargestellt.

\section{Ergebnisse}

\subsection{Deskriptive Ergebnisse}

Abbildung 1 gibt Aufschluss über die Verteilung der einzelnen Zielvariablen. Es zeigt sich eine große Ähnlichkeit in den Einstellungen zwischen türkischen Angehörigen der ersten und zweiten Generation, und ein deutlich abweichendes Muster bei den Deutschen. Markant ist dies für die eher allgemein gehaltene Aussage, für die Eltern Verantwortung zu übernehmen. 44 Prozent der türkischen Migranten beider Einwanderergenerationen stimmen stark zu, weniger als 10 Prozent sind unentschieden oder lehnen dies ab. Bei den Deutschen dagegen stimmt weniger als ein Fünftel dieser Aussage vorbehaltlos zu, und der Anteil der Personen, die unentschieden sind oder diese ablehnen, ist mit gut einem Fünftel deutlich größer.

Abbildung 1: Grad der Zustimmung zu den drei abhängigen Variablen, getrennt für die drei Untersuchungsgruppen (\%)

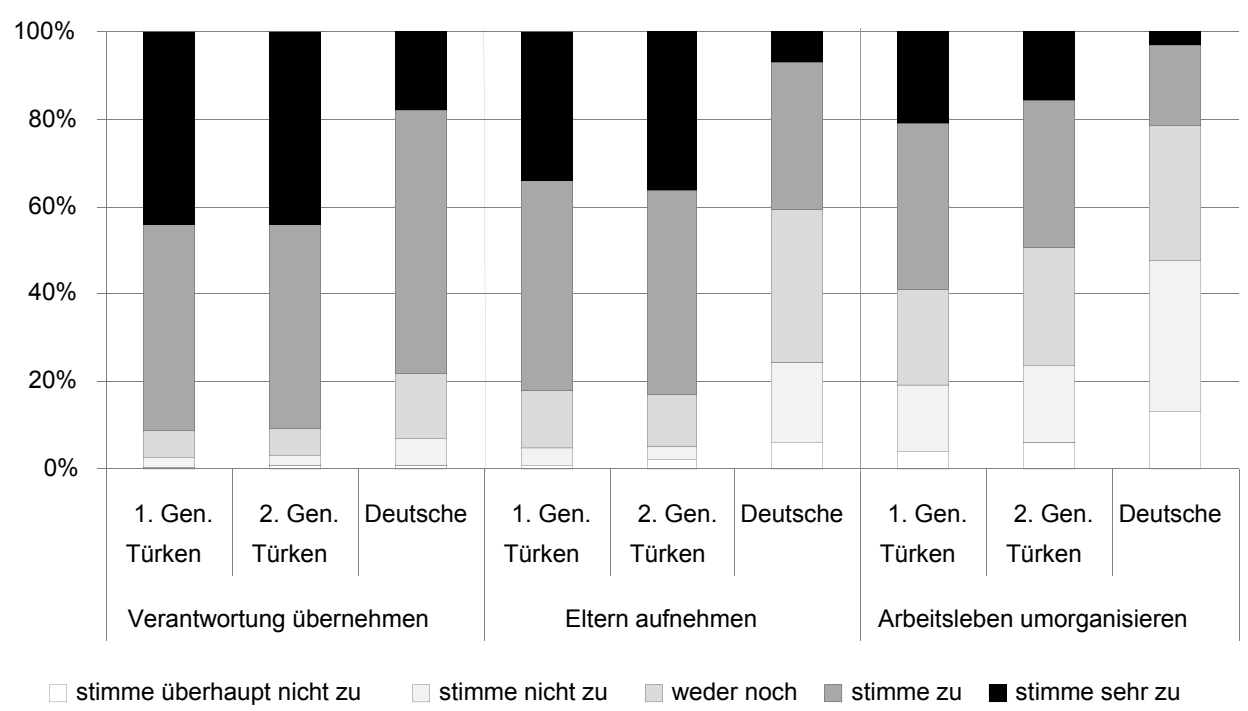

Quelle: Generations and Gender Survey 2005/2006, eigene Berechnungen. 
Ein sehr ähnliches Muster zeigt sich bei der eine konkrete Handlung beinhaltenden Aussage, wonach die Eltern im Bedarfsfall von den Kindern aufgenommen werden sollten (Kohabitation). Die Unterschiede zwischen den beiden Einwanderergenerationen sind marginal, mit deutlichen Abweichungen zu den Deutschen. In allen drei Gruppen ist die Zustimmung zurückhaltender, was vermutlich mit der spezifischer formulierten Verantwortungsübernahme zusammenhängt. So findet sich eine starke Zustimmung lediglich noch bei etwa einem Drittel der befragten türkischen Migranten (beider Gruppen). Allerdings liegt die Zustimmung insgesamt noch bei über 80 Prozent. Etwa ein Sechstel ist unentschieden oder lehnt dies ab. Die Zustimmung bei den Deutschen liegt lediglich bei etwa 40 Prozent. Beim dritten Item sinkt die Zustimmung weiter, in allen drei Gruppen. Deutlich weniger Befragte sehen die Aufgabe der Kinder darin, für die Eltern auch ihr Arbeitsleben umzuorganisieren, falls dies notwendig sein sollte. Bemerkenswert ist, dass sich hier die türkischen Migranten der zweiten Generation deutlicher von der Elterngeneration unterscheiden. Die Zustimmung liegt bei der ersten Generation noch bei etwa 60 Prozent, bei der zweiten nur noch bei 50 Prozent, mit geringeren Anteilen starker Zustimmung und einer stärkeren Ablehnung. Lediglich ein Fünftel der Deutschen würde dieser Aussage zustimmen, nur drei Prozent besonders stark.

Tabelle 1: Anteil der Zustimmung (nur: stimme [sehr] zu) nach Altersgruppen (\%)

\begin{tabular}{lccccccccccccc}
\hline & $\begin{array}{c}\text { Kinder sollten die Verant- } \\
\text { wortung für ihre Eltern } \\
\text { übernehmen, wenn diese } \\
\text { Hilfe brauchen. }\end{array}$ & $\begin{array}{c}\text { Kinder sollten ihre Eltern zu } \\
\text { sich nehmen, wenn diese nicht } \\
\text { mehr selbst für sich sorgen } \\
\text { können. }\end{array}$ & $\begin{array}{c}\text { Kinder sollten ihr Arbeitsleben } \\
\text { umorganisieren, um den } \\
\text { Bedürfnissen ihrer Eltern nach- } \\
\text { kommen zu können }\end{array}$ \\
$\begin{array}{l}\text { Altersgruppen } \\
\text { in Jahren }\end{array}$ & $<\mathbf{3 0}$ & $\mathbf{3 0 - 4 4}$ & $\mathbf{4 5 - 5 9}$ & $\mathbf{6 0 +}$ & $\mathbf{< 3 0}$ & $\mathbf{3 0 - 4 4}$ & $\mathbf{4 5 - 5 9}$ & $\mathbf{6 0 +}$ & $<\mathbf{3 0}$ & $\mathbf{3 0 - 4 4}$ & $\mathbf{4 5 - 5 9}$ & $\mathbf{6 0 +}$ \\
\hline Türken 1. Gen. & 92 & 93 & 89 & 90 & 85 & 81 & 81 & 82 & 60 & 56 & 59 & 64 \\
$\begin{array}{l}\text { Türken 2. Gen. } \\
\text { Deutsche }\end{array}$ & 92 & 90 & - & - & 85 & 78 & - & - & 51 & 46 & - & - \\
\hline
\end{tabular}

Quelle: Generations and Gender Survey 2005/2006, eigene Berechnungen.

In Tabelle 1 sind die Prozentwerte für die Zustimmung, sowohl die einfache als auch die besonders starke, abgetragen. Das beschriebene Muster gilt für die drei Gruppen der Deutschen und türkischen Migranten in allen Altersgruppen: Je globaler die Aussage, desto größer die Zustimmung. Die Koresidenz mit den Eltern wird stärker unterstützt als Veränderungen im Arbeitsleben, um den Eltern helfen zu können. Zugleich ähneln sich die türkischen Migranten der ersten und zweiten Generation stark in der Zustimmung zu den ersten beiden Aussagen, während sich deutliche Differenzen im letztgenannten Item zeigen. Die Unterschiede zu Deutschen sind in allen Altersgruppen groß. Zwischen den Altersgruppen zeigt sich tendenziell eine stärkere Zustimmung zu den Normen in der jüngsten und der höchsten Altersgruppe, dagegen geringere Werte in den beiden mittleren Altersgruppen. In der türkischen zweiten Generation nimmt die Zustimmung zwischen der jüngsten und mittleren Altersgruppe deutlich ab. Diese Verteilungen legen nahe, dass Personen, die bereits stärker in der Verantwortung für ihre Eltern stehen bzw. mit den Herausforderung der Hilfe für die und Pflege der Eltern konkreter konfrontiert sind, die Rolle der Familie geringer einschätzen (wollen), möglicherweise aufgrund anderer Verantwortlichkeiten im Berufs- und Familienleben. Im Gegenzug dazu stehen Personen, für die diese Normen noch ein relativ abstrakter Gedanke sind (Jüngere) bzw. diejenigen, die 
selbst bereits oder bald auf eben diese intergenerationale Unterstützung angewiesen sind. Die geringere Zustimmung von Älteren zu den spezifischeren Aufgaben von Kindern zeigt dabei zugleich, dass Ältere (sowohl Deutsche als auch Türken) die Herausforderungen, vor denen die Kinder im Bedarfsfall stehen, sehr wohl wahrnehmen und ihnen diese vielfach nicht aufbürden wollen. Insgesamt bestätigen die Befunde allerdings die deutlich größere Familienorientierung bei türkischen Migranten (H1), mit gewissen, aber tendenziell geringen Unterschieden zwischen der ersten und zweiten Generation (H2).

Tabelle 2: Beschreibung der Einflussfaktoren für die Untersuchungsgruppen (\%)

\begin{tabular}{|c|c|c|c|}
\hline & Türken 1. Gen & Türken 2. Gen & Deutsche \\
\hline Alter (in Jahren) & 41 Jahre $(12,5)$ & 27 Jahre $(5,7)$ & 49 Jahre $(16,1)$ \\
\hline \multicolumn{4}{|l|}{ Geschlecht } \\
\hline Mann & 52,7 & 52,5 & 46,2 \\
\hline \multicolumn{4}{|l|}{ Bildung } \\
\hline niedrig & 65,9 & 43,6 & 12,6 \\
\hline mittel/hoch & 34,2 & 56,4 & 87,4 \\
\hline \multicolumn{4}{|l|}{ Partnerschaftsstatus } \\
\hline mit Partner & 84,2 & 57,4 & 72,0 \\
\hline \multicolumn{4}{|l|}{ Kinder } \\
\hline mind. ein Kind & 91,0 & 85,4 & 72,5 \\
\hline \multicolumn{4}{|l|}{ Gesundheit } \\
\hline gut & 74,2 & 92,3 & 73,4 \\
\hline eher schlecht & 25,2 & 7,4 & 26,3 \\
\hline keine Angabe & 0,6 & 0,3 & 0,3 \\
\hline \multicolumn{4}{|c|}{ Teilnahme an religiösen Veranstaltungen } \\
\hline mehrmals pro Woche & 10,0 & 5,5 & 1,5 \\
\hline mehrmals im Monat & 27,5 & 16,6 & 12,4 \\
\hline mehrmals im Jahr & 21,9 & 24,2 & 25,4 \\
\hline seltener & 16,6 & 25,9 & 27,4 \\
\hline nie & 22,8 & 25,7 & 32,7 \\
\hline keine Angabe & 1,2 & 2,3 & 0,6 \\
\hline \multicolumn{4}{|l|}{ Einsamkeit: } \\
\hline trifft zu & 25,1 & 24,7 & 17,4 \\
\hline$n$ & 2972 & 935 & 7705 \\
\hline
\end{tabular}

Notiz: Chi-Quadrat-Tests zwischen den abhängigen Variablen der 1. und 2. Generation bzw. der deutschen Untersuchungsgruppe und den unabhängigen Variablen erbrachten für alle Variablen höchst signifikante Ergebnisse $(\mathrm{p}<0,01)$.

Quelle: Generations and Gender Survey 2005/2006, eigene Berechnungen

Tabelle 2 zeigt die in den folgenden Analysen berücksichtigten Prädiktoren für die drei Gruppen. Deutlich sind die Differenzen im Durchschnittsalter. Das deutlich jüngere Alter der türkischen Migranten der zweiten Generation spiegelt sich in anderen Merkmalen (bspw. im Partnerschaftsstatus und bei der Gesundheit) wider. Während die Geschlechteranteile in beiden Gruppen türkischer Migranten gleich sind, ist der Frauenanteil bei den Deutschen größer. Das Bildungsniveau ist bei türkischen Migranten der ersten Generation am geringsten und bei Deutschen am höchsten. Umgekehrt ist die Kinderlosigkeit bei 
letzteren mit etwa 27 Prozent am höchsten, während nur 9 Prozent der türkischen Migranten der ersten Generation keine Kinder haben. Diese Gruppe nimmt auch am häufigsten an religiösen Veranstaltungen teil und fühlt sich zugleich deutlich häufiger als Deutsche, aber ähnlich häufig wie Angehörige der zweiten Generation, einsam. Im Folgenden soll untersucht werden, inwiefern diese Differenzen die Unterschiede in den Einstellungsmustern (mit)erklären können.

\subsection{Multivariate Analyse}

In Tabelle 3 sind jeweils zwei Regressionsmodelle je Einstellungsvariable abgebildet. Das erste Modell (M1) beinhaltet die zentrale Einflussvariable Herkunft/Migrationsstatus, das zweite Modell (M2) berücksichtigt zusätzlich die weiteren Prädiktoren. Die multivariaten Analysen bestätigen die Differenzen zwischen den drei Herkunfts-/Migrantengruppen. Die Antwort auf die Frage, ob die soziodemographischen und sozioökonomischen Unterschiede die oben konstatierten Differenzen zwischen Migranten, ihren Nachkommen und der einheimischen deutschen Bevölkerung erklären können, lautet: Sie können es kaum. Die Differenzen zwischen Deutschen einerseits, und Migranten der ersten bzw. zweiten Generationen andererseits, bleiben hoch signifikant im multivariaten Modell bestehen $(\mathrm{p}<0,001)$. Die Koeffizienten verändern sich nur leicht zwischen den Modellen 1 und 2. Dabei sind die Koeffizienten bei den türkischen Migranten der zweiten Generation für die Einstellungsitems „Verantwortung übernehmen“ und „Arbeitsleben umgestalten“ jeweils etwas niedriger, für das Item „Eltern aufnehmen“ unterscheiden sich Angehörige der zweiten Generation dagegen noch stärker von den Deutschen als Migranten der ersten Generation. In alternativen Modellen, in denen die zweite Generation als Referenzkategorie definiert war (hier nicht dargestellt), zeigten sich durchgängig hochsignifikante Differenzen zu Deutschen. Die Unterschiede zur ersten Generation waren insignifikant, mit Ausnahme des Einstellungsitems „Arbeitsleben umgestalten“ (Odds Ratio für die erste Generation: $1,35 \mathrm{p}<0,001)$. Das bedeutet umgekehrt, dass bei den türkischen Migranten der zweiten Generation die Erwartung, dass Kinder ihr Arbeitsleben umorganisieren, bereits signifikant kleiner ist als noch in der ersten Generation.

Diese Befunde lassen auf starke ethnisch-kulturell bedingte Unterschiede in den Werteinstellungen schließen, die sich in der Folgegeneration weitgehend fortsetzen. Zumindest im Bereich der Werte zur familialen Solidarität finden sich weniger Anzeichen von Akkulturation als von Transmission. 
Tabelle 3: Logistische Regressionsmodelle (OR) zur Zustimmung hinsichtlich zukünftiger Hilfeleistungen/Pflegeübernahme der Eltern

\begin{tabular}{|c|c|c|c|c|c|c|}
\hline & \multicolumn{2}{|c|}{$\begin{array}{l}\text { Kinder sollten die Ver- } \\
\text { antwortung für Eltern } \\
\text { übernehmen, wenn diese } \\
\text { Hilfe brauchen. }\end{array}$} & \multicolumn{2}{|c|}{$\begin{array}{l}\text { Kinder sollten Eltern zu } \\
\text { sich nehmen, wenn diese } \\
\text { nicht mehr selbst für } \\
\text { sich sorgen können. }\end{array}$} & \multicolumn{2}{|c|}{$\begin{array}{l}\text { Kinder sollten Arbeits- } \\
\text { leben umorganisieren, } \\
\text { um den Bedürfnissen } \\
\text { ihrer Eltern nach- } \\
\text { kommen zu können }\end{array}$} \\
\hline & M1 & M2 & M1 & M2 & M1 & M2 \\
\hline \multicolumn{7}{|c|}{ Migrationsstatus (Ref.: Deutsche) } \\
\hline Türken 1. Generation & $\begin{array}{l}3,11^{* * *} \\
(0,22)\end{array}$ & $\begin{array}{l}2,69^{\star * \star} \\
(0,23)\end{array}$ & $\begin{array}{l}6,53^{* * *} \\
(0,34)\end{array}$ & $\begin{array}{l}4,52^{\star * *} \\
(0,28)\end{array}$ & $\begin{array}{l}4,99^{* * *} \\
(0,23)\end{array}$ & $\begin{array}{l}4,09^{\star \star \star} \\
(0,24)\end{array}$ \\
\hline Türken 2. Generation & $\begin{array}{l}2,86^{* * *} \\
(0,33)\end{array}$ & $\begin{array}{l}2,64^{\star * *} \\
(0,33)\end{array}$ & $\begin{array}{l}6,90^{\star \star \star} \\
(0,61)\end{array}$ & $\begin{array}{l}5,02^{\star \star *} \\
(0,49)\end{array}$ & $\begin{array}{l}3,53^{\star * *} \\
(0,25)\end{array}$ & $\begin{array}{l}3,04^{\star \star *} \\
(0,25)\end{array}$ \\
\hline Alter & & $\begin{array}{l}1,00 \\
(0,00)\end{array}$ & & $\begin{array}{l}0,99^{* * *} \\
(0,00)\end{array}$ & & $\begin{array}{l}1,00 \\
(0,00)\end{array}$ \\
\hline \multicolumn{7}{|l|}{ Geschlecht (Ref.: Frau) } \\
\hline Mann & & $\begin{array}{l}1,20^{* \star *} \\
(0,06)\end{array}$ & & $\begin{array}{l}1,13^{\star *} \\
(0,05)\end{array}$ & & $\begin{array}{l}1,12^{\star *} \\
(0,05)\end{array}$ \\
\hline \multicolumn{7}{|l|}{$\begin{array}{l}\text { Partnerschaftsstatus } \\
\text { (Ref.: ohne Partner) }\end{array}$} \\
\hline mit Partner & & $\begin{array}{l}0,96 \\
(0,07)\end{array}$ & & $\begin{array}{l}0,88^{*} \\
(0,05)\end{array}$ & & $\begin{array}{l}0,93 \\
(0,06)\end{array}$ \\
\hline \multicolumn{7}{|l|}{ Bildung (Ref.: niedrig) } \\
\hline mittel/hoch & & $\begin{array}{l}0,82^{* *} \\
(0,06)\end{array}$ & & $\begin{array}{l}0,67^{* * *} \\
(0,04)\end{array}$ & & $\begin{array}{l}0,81^{* * *} \\
(0,04)\end{array}$ \\
\hline \multicolumn{7}{|c|}{ Kinder (Ref.: mind. ein Kind) } \\
\hline keine Kinder & & $\begin{array}{l}1,18^{*} \\
(0,09)\end{array}$ & & $\begin{array}{l}0,94 \\
(0,06)\end{array}$ & & $\begin{array}{l}1,09 \\
(0,07)\end{array}$ \\
\hline \multicolumn{7}{|l|}{ Gesundheit (Ref.: gut) } \\
\hline eher schlecht & & $\begin{array}{l}0,81^{* *} \\
(0,05)\end{array}$ & & $\begin{array}{l}1,04 \\
(0,05)\end{array}$ & & $\begin{array}{l}1,05 \\
(0,06)\end{array}$ \\
\hline keine Angabe & & $\begin{array}{l}0,61 \\
(0,23)\end{array}$ & & $\begin{array}{l}1,69 \\
(0,63)\end{array}$ & & $\begin{array}{l}0,90 \\
(0,31)\end{array}$ \\
\hline \multicolumn{7}{|c|}{$\begin{array}{l}\text { Teilnahme an religiösen Veranstal- } \\
\text { tungen (Ref.: mehrmals pro Woche) }\end{array}$} \\
\hline mehrmals im Monat & & $\begin{array}{l}0,94 \\
(0,17)\end{array}$ & & $\begin{array}{l}0,92 \\
(0,12)\end{array}$ & & $\begin{array}{l}0,88 \\
(0,10)\end{array}$ \\
\hline mehrmals im Jahr & & $\begin{array}{l}0,79 \\
(0,14)\end{array}$ & & $\begin{array}{l}0,67^{\star *} \\
(0,09)\end{array}$ & & $\begin{array}{l}0,79^{*} \\
(0,09)\end{array}$ \\
\hline seltener & & $\begin{array}{l}0,68^{*} \\
(0,12)\end{array}$ & & $\begin{array}{l}0,58^{\star * *} \\
(0,08)\end{array}$ & & $\begin{array}{l}0,63^{\star * *} \\
(0,07)\end{array}$ \\
\hline nie & & $\begin{array}{l}0,64^{\star *} \\
(0,11)\end{array}$ & & $\begin{array}{l}0,50^{\star * *} \\
(0,06)\end{array}$ & & $\begin{array}{l}0,57^{\star \star \star} \\
(0,06)\end{array}$ \\
\hline keine Angabe & & $\begin{array}{l}0,41^{* *} \\
(0,12)\end{array}$ & & $\begin{array}{l}0,43^{\star *} \\
(0,11)\end{array}$ & & $\begin{array}{l}0,96 \\
(0,23)\end{array}$ \\
\hline \multicolumn{7}{|c|}{ Einsamkeit (Ref.: trifft nicht zu) } \\
\hline trifft zu & & $\begin{array}{l}1,19^{*} \\
(0,08)\end{array}$ & & $\begin{array}{l}1,24^{* * *} \\
(0,07)\end{array}$ & & $\begin{array}{l}1,26^{\star \star *} \\
(0,07)\end{array}$ \\
\hline $\mathrm{n}$ & 11612 & 11612 & 11612 & 11612 & 11612 & 11612 \\
\hline Pseudo R² (McFadden) & 0,03 & 0,04 & 0,12 & 0,13 & 0,09 & 0,10 \\
\hline LR chi ${ }^{2}$ & 367,54 & 448,95 & 1902,20 & 2099,89 & 1369,77 & 1489,76 \\
\hline
\end{tabular}

Quelle: Generations and Gender Survey 2005/2006, eigene Berechnungen.

Notiz: Signifikanzniveau $\left({ }^{\circ} \mathrm{p}<0,10, * \mathrm{p}<0,05, * * \mathrm{p}<0,01, * * * \mathrm{p}<0,001\right)$. 
Dabei ist die Zustimmung zu den drei Einstellungsitems grundsätzlich höher bei Männern als bei Frauen und das Bildungsniveau zeigt den erwarteten negativen Effekt, wonach eine höhere Bildung die Wahrscheinlichkeit einer Zustimmung reduziert. Der Partnerschaftsstatus ist von geringer Bedeutung, zeigt aber insgesamt in die postulierte Richtung: Wenn ein Partner vorhanden ist, ist die Wahrscheinlichkeit, Erwartungen an Kinder zu haben, geringer. Wer selbst (noch) kein Kind hat, erwartet eher, dass sich Kinder um ihre bedürftigen Eltern kümmern. Allerdings verschwindet dieser Effekt, wenn es um konkretere Hilfeerwartungen geht. Unerwartet ist die Richtung des Einflusses des eigenen Gesundheitsstatus: Bei schlechter Gesundheit ist die Wahrscheinlichkeit einer Zustimmung geringer. Hier wird zu überprüfen sein, ob sich dieser Effekt auch in nach Altersgruppen getrennten Analysen hält, in denen durch die Altersgruppierung Geber und Empfänger potenzieller Hilfe getrennt betrachtet werden (siehe unten). Hinsichtlich des Einflusses der Teilnahme an religiösen Aktivitäten kann nicht die Hypothese des alternativen Netzwerks, aber die Familialismusannahme bestätigt werden: Über religiöse Aktivitäten wenig eingebundene Personen haben zugleich eine geringe Wahrscheinlichkeit, den Solidaritätsnormen zuzustimmen. Es sind also eher die Personen, die in religiöse Netzwerke eingebunden und vermutlich entsprechend religiös orientiert sind, die mit einer größeren Wahrscheinlichkeit entsprechende intergenerationale Solidaritätserwartungen zum Ausdruck bringen. Zugleich erhöhen Einsamkeitsgefühle die Wahrscheinlichkeit der Zustimmung: Wer sich wenig eingebunden fühlt, hat höhere Erwartungen an die Familie.

Das Alter spielte in den obigen Modellen nur eine geringe Rolle. Um die Bedeutung des Alters bzw. der jeweiligen Phase des Lebenslaufs zu erfassen und etwaige Effekte als potenzielle Geber und Empfänger von Hilfe kontrollieren zu können, werden im Folgenden die Modelle für jeweils vier Altersgruppen berechnet. Finden sich ähnliche Effekte in allen Lebensphasen, existieren in allen Altersgruppen also ähnlich große Differenzen zwischen Deutschen und Türkeistämmigen? Wie angedeutet könnten wir erwarten, dass Differenzen mit dem Alter zunehmen, wenn zum Beispiel die eigene Bedarfslage Unterstützung eher erforderlich macht und dann herkunftsbedingte Normen an Bedeutung gewinnen, auch im Sinne eines ,ethnic revival“ (Dietzel-Papakyriakou 1993). Umgekehrt wäre zu erwarten, dass Jüngere, die noch nicht mit einer entsprechenden eigenen oder elterlichen Bedarfssituation konfrontiert sind, diesem Thema indifferenter und damit insgesamt ähnlicher (weil unabhängiger von eigenen kulturellen Normen) gegenüberstehen. Die deskriptiven Befunde (siehe oben) wiesen auf beständige Differenzen hin, die hier im Hinblick auf mögliche Kompositionseffekte aufgrund unterschiedlicher soziodemographischer und -ökonomischer Merkmale untersucht werden.

Tatsächlich zeigt sich auch in den Tabellen 4a bis 4c für alle Altersgruppen das oben für die Gesamtpopulation beschriebene Muster. Die Differenzen zwischen Deutschen einerseits und türkischen Migranten andererseits bleiben in allen Altersgruppen, auch bei Kontrolle weiterer Variablen, hochsignifikant bestehen. Bemerkenswert ist wiederum, dass auch hier nur ein sehr geringer Einfluss von den getesteten Prädiktoren ausgeht. Die ethnisch-kulturellen Unterschiede, die sich in den Werteinstellungen zeigen, existieren beständig über die Einwanderergenerationen, unabhängig von Alter und Lebensphase und anderen Eigenschaften. 
Tabelle 4a: Logistische Regression (OR) zur Zustimmung hinsichtlich der abhängigen Variable „Verantwortung übernehmen“ nach Alter

\begin{tabular}{|c|c|c|c|c|}
\hline & $<30$ Jahre & 30-44 Jahre & 45-59 Jahre & $\begin{array}{l}60 \text { Jahre } \\
\text { und älter }\end{array}$ \\
\hline \multicolumn{5}{|c|}{ Migrationsstatus (Ref.: Deutsche) } \\
\hline Türken 1. Generation & $\begin{array}{l}2,35^{\star * *} \\
(0,47)\end{array}$ & $\begin{array}{l}4,24^{\star * *} \\
(0,58)\end{array}$ & $\begin{array}{l}2,62^{\star \star *} \\
(0,45)\end{array}$ & $\begin{array}{l}1,89^{* *} \\
(0,41)\end{array}$ \\
\hline Türken 2. Generation & $\begin{array}{l}2,19^{* * *} \\
(0,39)\end{array}$ & $\begin{array}{l}2,91^{* * *} \\
(0,58)\end{array}$ & - & - \\
\hline \multicolumn{5}{|l|}{ Geschlecht } \\
\hline Mann (Ref.: Frau) & $\begin{array}{l}1,03 \\
(0,14)\end{array}$ & $\begin{array}{l}1,17^{\circ} \\
(0,11)\end{array}$ & $\begin{array}{l}1,27^{*} \\
(0,12)\end{array}$ & $\begin{array}{l}1,06 \\
(0,12)\end{array}$ \\
\hline \multicolumn{5}{|c|}{ Partnerschaftsstatus (Ref.: ohne Partner) } \\
\hline mit Partner & $\begin{array}{l}0,92 \\
(0,13)\end{array}$ & $\begin{array}{l}1,14 \\
(0,14)\end{array}$ & $\begin{array}{l}1,04 \\
(0,15)\end{array}$ & $\begin{array}{l}0,59^{*} \\
(0,14)\end{array}$ \\
\hline \multicolumn{5}{|l|}{ Bildung (Ref.: niedrig) } \\
\hline mittel/hoch & $\begin{array}{l}1,03 \\
(0,15)\end{array}$ & $\begin{array}{l}0,93 \\
(0,13)\end{array}$ & $\begin{array}{l}0,84 \\
(0,14)\end{array}$ & $\begin{array}{l}0,76^{\circ} \\
(0,11)\end{array}$ \\
\hline \multicolumn{5}{|c|}{ Kinder (Ref.: mind. ein Kind) } \\
\hline keine Kinder & $\begin{array}{l}1,03 \\
(0,17)\end{array}$ & $\begin{array}{l}1,21 \\
(0,17)\end{array}$ & $\begin{array}{l}1,02 \\
(0,16)\end{array}$ & $\begin{array}{l}0,73 \\
(0,16)\end{array}$ \\
\hline \multicolumn{5}{|l|}{ Gesundheit (Ref.: gut) } \\
\hline eher schlecht & $\begin{array}{l}0,68 \\
(0,17)\end{array}$ & $\begin{array}{l}0,71^{\star *} \\
(0,08)\end{array}$ & $\begin{array}{l}0,79^{*} \\
(0,08)\end{array}$ & $\begin{array}{l}0,96 \\
(0,10)\end{array}$ \\
\hline keine Angabe & $\begin{array}{l}0,50 \\
(0,56)\end{array}$ & $\begin{array}{l}1,22 \\
(0,95) \\
\end{array}$ & $\begin{array}{l}0,27^{\star} \\
(0,18)\end{array}$ & $\begin{array}{l}0,71 \\
(0,56) \\
\end{array}$ \\
\hline$n$ & 2166 & 3980 & 2769 & 2693 \\
\hline Pseudo $\mathrm{R}^{2}$ (McFadden) & 0,03 & 0,07 & 0,03 & 0,02 \\
\hline LR chi & 41,93 & 249,48 & 85,68 & 39,20 \\
\hline
\end{tabular}

Notiz: Signifikanzniveau $\left({ }^{\circ} \mathrm{p}<0,10, * \mathrm{p}<0,05, * * \mathrm{p}<0,01, * * * \mathrm{p}<0,001\right)$.

Quelle: Generations and Gender Survey 2005/2006, eigene Berechnungen.

Dabei spielt im Hinblick auf das globale Item der Verantwortungsübernahme (Tabelle 4a) die eigene gesundheitliche Situation eine Rolle, allerdings nur in den mittleren Altersgruppen, also bei den Kindern, die als Unterstützer selbst gefordert sind bzw. wären: Ist der eigene Gesundheitszustand schlecht, so findet sich eine geringere Wahrscheinlichkeit für eine Zustimmung - möglicherweise, weil die eigenen (gesundheitlichen) Ressourcen fehlen, um den eigenen Eltern selbst umfassend helfen zu können.

Nicht die Gesundheit, sondern das Vorhandensein eines Partners und das Bildungsniveau sind bedeutsam für die Zustimmung zu der Norm, als Kind die Eltern bei sich aufzunehmen bzw. das Arbeitsleben umzuorganisieren. Der Partner ist offensichtlich die zentrale Alternative: Nur wer allein ist, hat mit einer höheren Wahrscheinlichkeit Erwartungen an Kinder. Bei den Älteren über 60 Jahre zeigen sich Bestrebungen nach Selbständigkeit oder alternative Unterstützungsformen in Abhängigkeit von der Bildung: Je höher die Bildung, desto geringer die Wahrscheinlichkeit hoher Erwartungen an Kinder. Dieser Bildungseffekt zeigt sich tendenziell in allen drei Items. 
Tabelle 4b: Logistische Regression (OR) zur Zustimmung hinsichtlich der abhängigen Variable „Eltern aufnehmen“ nach Alter

\begin{tabular}{|c|c|c|c|c|}
\hline & $<30$ Jahre & 30-44 Jahre & 45-59 Jahre & $\begin{array}{l}60 \text { Jahre } \\
\text { und älter }\end{array}$ \\
\hline \multicolumn{5}{|c|}{ Migrationsstatus (Ref.: Deutsche) } \\
\hline Türken 1. Generation & $\begin{array}{l}4,40^{* \star *} \\
(0,64)\end{array}$ & $\begin{array}{l}6,27^{\star * *} \\
(0,63)\end{array}$ & $\begin{array}{l}5,37^{\star * *} \\
(0,74)\end{array}$ & $\begin{array}{l}4,46^{* * *} \\
(0,73)\end{array}$ \\
\hline Türken 2. Generation & $\begin{array}{l}5,03^{* * *} \\
(0,67)\end{array}$ & $\begin{array}{l}5,44^{* * *} \\
(0,81)\end{array}$ & - & - \\
\hline \multicolumn{5}{|l|}{ Geschlecht (Ref.: Frau) } \\
\hline Mann & $\begin{array}{l}0,94 \\
(0,10)\end{array}$ & $\begin{array}{l}1.06 \\
(0,08)\end{array}$ & $\begin{array}{l}1,22^{*} \\
(0,10)\end{array}$ & $\begin{array}{l}1,19^{*} \\
(0,11)\end{array}$ \\
\hline \multicolumn{5}{|c|}{ Partnerschaftsstatus (Ref.: ohne Partner) } \\
\hline mit Partner & $\begin{array}{l}1,19 \\
(0,13)\end{array}$ & $\begin{array}{l}0,80^{*} \\
(0,09)\end{array}$ & $\begin{array}{l}1,10 \\
(0,14)\end{array}$ & $\begin{array}{l}0,46^{* * *} \\
(0,09)\end{array}$ \\
\hline \multicolumn{5}{|l|}{ Bildung (Ref.: niedrig) } \\
\hline mittel/hoch & $\begin{array}{l}0,73^{\star *} \\
(0,08)\end{array}$ & $\begin{array}{l}0,89 \\
(0,09)\end{array}$ & $\begin{array}{l}0,64^{* *} \\
(0,09)\end{array}$ & $\begin{array}{l}0,56^{\star * *} \\
(0,06)\end{array}$ \\
\hline \multicolumn{5}{|c|}{ Kinder (Ref.: mind. ein Kind) } \\
\hline keine Kinder & $\begin{array}{l}0,82^{\circ} \\
(0,10)\end{array}$ & $\begin{array}{l}0,75^{\star} \\
(0,09)\end{array}$ & $\begin{array}{l}1,09 \\
(0,15)\end{array}$ & $\begin{array}{l}0,55^{\star *} \\
(0,10)\end{array}$ \\
\hline \multicolumn{5}{|l|}{ Gesundheit (Ref.: gut) } \\
\hline eher schlecht & $\begin{array}{l}0,95 \\
(0,20)\end{array}$ & $\begin{array}{l}0,88 \\
(0,09)\end{array}$ & $\begin{array}{l}0,99 \\
(0,09)\end{array}$ & $\begin{array}{l}1,10 \\
(0,09)\end{array}$ \\
\hline keine Angabe & $\begin{array}{l}1,83 \\
(2,19)\end{array}$ & $\begin{array}{l}3,06^{\circ} \\
(2,12)\end{array}$ & $\begin{array}{l}1,31 \\
(0,88) \\
\end{array}$ & $\begin{array}{l}0,98 \\
(0,67)\end{array}$ \\
\hline$n$ & 2166 & 3980 & 2769 & 2693 \\
\hline Pseudo R² (McFadden) & 0,12 & 0,14 & 0,11 & 0,07 \\
\hline LR chi ${ }^{2}$ & 323,07 & 776,34 & 421,83 & 264,36 \\
\hline
\end{tabular}

Notiz: Signifikanzniveau $\left({ }^{\circ} \mathrm{p}<0,10,{ }^{*} \mathrm{p}<0,05, * * \mathrm{p}<0,01, * * * \mathrm{p}<0,001\right)$.

Quelle: Generations and Gender Survey 2005/2006, eigene Berechnungen. 
Tabelle 4c: Logistische Regression (OR) zur Zustimmung hinsichtlich der abhängigen Variable „Arbeitsleben umorganisieren“ nach Alter

\begin{tabular}{|c|c|c|c|c|}
\hline & $<30$ Jahre & 30-44 Jahre & 45-59 Jahre & $\begin{array}{l}60 \text { Jahre } \\
\text { und älter }\end{array}$ \\
\hline \multicolumn{5}{|c|}{ Mirgrationsstatus (Ref.: Deutsche) } \\
\hline Türken 1. Generation & $\begin{array}{l}4,24^{* * *} \\
(0,53)\end{array}$ & $\begin{array}{l}4,08^{* * *} \\
(0,37)\end{array}$ & $\begin{array}{l}5,54^{* * *} \\
(0,74)\end{array}$ & $\begin{array}{l}4,99^{* * *} \\
(0,73)\end{array}$ \\
\hline Türken 2. Generation & $\begin{array}{l}3,08^{* * *} \\
(0,35)\end{array}$ & $\begin{array}{l}3,00^{* * *} \\
(0,39)\end{array}$ & - & - \\
\hline \multicolumn{5}{|l|}{ Geschlecht (Ref.: Frau) } \\
\hline Mann & $\begin{array}{l}0,97 \\
(0,09)\end{array}$ & $\begin{array}{l}1,15^{\circ} \\
(0,09)\end{array}$ & $\begin{array}{l}1,15 \\
(0,11)\end{array}$ & $\begin{array}{l}1,26^{*} \\
(0,12)\end{array}$ \\
\hline \multicolumn{5}{|l|}{$\begin{array}{l}\text { Partnerschaftsstatus } \\
\text { (Ref.: ohne Partner) }\end{array}$} \\
\hline mit Partner & $\begin{array}{l}1,03 \\
(0,10)\end{array}$ & $\begin{array}{l}1,15 \\
(0,13)\end{array}$ & $\begin{array}{l}0,82 \\
(0,12)\end{array}$ & $\begin{array}{l}0,61^{*} \\
(0,12)\end{array}$ \\
\hline \multicolumn{5}{|l|}{ Bildung (Ref.: niedrig) } \\
\hline mittel/hoch & $\begin{array}{l}0,91 \\
(0,09)\end{array}$ & $\begin{array}{l}0,79^{*} \\
(0,07)\end{array}$ & $\begin{array}{l}0,93 \\
(0,13)\end{array}$ & $\begin{array}{l}0,71^{* *} \\
(0,08)\end{array}$ \\
\hline \multicolumn{5}{|c|}{ Kinder (Ref.: mind. ein Kind) } \\
\hline keine Kinder & $\begin{array}{l}1,03 \\
(0,12)\end{array}$ & $\begin{array}{l}0,87 \\
(0,11)\end{array}$ & $\begin{array}{l}0,99 \\
(0,15)\end{array}$ & $\begin{array}{l}0,90 \\
(0,18)\end{array}$ \\
\hline \multicolumn{5}{|l|}{ Gesundheit (Ref.: gut) } \\
\hline eher schlecht & $\begin{array}{l}0,99 \\
(0,19)\end{array}$ & $\begin{array}{l}0,96 \\
(0,10)\end{array}$ & $\begin{array}{l}1,07 \\
(0,11)\end{array}$ & $\begin{array}{l}1,05 \\
(0,10)\end{array}$ \\
\hline keine Angabe & $\begin{array}{l}0,31 \\
(0,35)\end{array}$ & $\begin{array}{l}0,77 \\
(0,45)\end{array}$ & $\begin{array}{l}1,20 \\
(0,90)\end{array}$ & $\begin{array}{l}1,56 \\
(1,00) \\
\end{array}$ \\
\hline $\mathrm{n}$ & 2166 & 3980 & 2769 & 2693 \\
\hline Pseudo $\mathrm{R}^{2}$ (McFadden) & 0,07 & 0,10 & 0,11 & 0,08 \\
\hline LR chi² & 213,00 & 509,53 & 356,32 & 255,89 \\
\hline
\end{tabular}

Notiz: Signifikanzniveau $\left({ }^{\circ} \mathrm{p}<0,10,{ }^{*} \mathrm{p}<0,05, * * \mathrm{p}<0,01, * * * \mathrm{p}<0,001\right)$.

Quelle: Generations and Gender Survey 2005/2006, eigene Berechnungen.

\section{Zusammenfassung und Diskussion}

Mit Blick auf die Alterung der ersten Generation türkischer Einwanderer und dem damit steigenden Pflegebedarf stellt sich die Frage nach den familialen Solidaritätspotenzialen. In diesem Beitrag haben wir die Einstellungen zur Hilfe an und Pflege der Eltern durch Kinder in den Mittelpunkt gestellt und untersucht, inwiefern sich diese von jenen der einheimischen deutschen Bevölkerung unterscheiden. Dabei interessierte v.a. die Rolle ethnisch-kultureller gegenüber soziodemographischen und sozioökonomischen Einflussfaktoren. Außerdem sollte untersucht werden, ob sich Unterschiede zu Deutschen in nachfolgenden Generationen fortsetzen und sich in verschiedenen Altersgruppen und Lebensphasen ähnlich unterschiedliche Muster zeigen. Vor dem Hintergrund der unterschiedlichen „Familienkulturen“ in den Herkunfts- und Zielländern Türkei und Deutschland (Kağıtçıbaş1 1996; Ataca 2006; Nauck/Suckow 2006) geben die Befunde Aufschluss über kulturelle Transmissions- und Akkulturationsprozesse in der Migration. Zugleich 
zeigen sie, wie es um die familialen Solidaritätspotenziale in den von Alterungsprozessen ebenso wie die einheimische deutsche Bevölkerung betroffenen türkischen Migrantenfamilien bestellt ist: Wollen die Jüngeren die Unterstützungsleistung der Pflege in der Familie noch übernehmen bzw. wollen die Älteren diese Unterstützungsleistung durch ihre Kinder noch in Anspruch nehmen? Die Forschungslage zu diesem Aspekt der Generationenbeziehungen in Migrantenfamilien ist in Deutschland bisher noch dürftig, besonders zu Einstellungsunterschieden zwischen den Generationen ist bislang im Rahmen quantitativer Studien wenig geforscht worden (Vogel 2012; Zimmermann 2012).

Die Befunde dieser Analysen, wonach sich türkische Migranten und einheimische Deutsche sowohl in der ersten als auch in der zweiten Generation deutlich in den Einstellungen zur Generationensolidarität unterscheiden, bestätigen die Hypothesen 1 und 2 sowie ähnliche Analysen in den Niederlanden (de Valk/Schans 2008, Arends-Toth/Vijver 2008). In den Einstellungsdifferenzen, die über alle Altersgruppen bzw. Geburtskohorten hinweg bestehen (Bestätigung der Hypothesen $3 \mathrm{a}, \mathrm{b}$ und c), zeigen sich demnach auch in Deutschland ethnisch-kulturelle Muster, die nur in geringem Maße durch sozialstrukturelle Merkmale beeinflusst sind. Da für die Analysen Querschnittsdaten verwendet wurden, kann hier keine abschließende Aussage zur Bedeutung von Alters- versus Kohorteneffekten gemacht werden. Die (wenigen) konstatierten altersspezifischen Unterschiede können auf das Alter bzw. die Lebenslaufphase zurückgeführt werden, aber auch Ausdruck von (geburts-)kohortenspezifischen Einstellungsmustern sein.

Insgesamt machen die Daten deutlich, dass junge Menschen ihre Unterstützungsverpflichtung für die Elterngeneration antizipieren und Eltern ihrerseits entsprechende Hilfeleistungen erwarten. Bemerkenswert ist, dass der Grad der Zustimmung sowohl bei Deutschen als auch bei türkischen Migranten - mit deutlichen Niveaudifferenzen - besonders hoch ist, wenn global nach der Verantwortung für die hilfebedürftigen Eltern gefragt wird. Deutlich geringer ist sie jedoch, wenn es um konkrete Handlungen der Kinder geht, die das bisherige Leben der Kinder stark einschränken würden. Vor allem berufliche Veränderungen wollen weder Deutsche noch türkeistämmige Migranten - wiederum mit klaren Niveaudifferenzen - den Kindern zumuten, über alle Altersgruppen hinweg. Damit werden zum einen ökonomische Notwendigkeiten anerkannt (Einkommensverluste sollen verhindert werden), aber auch die mit einer Berufstätigkeit einhergehenden nicht-ökonomischen Ressourcen wertgeschätzt, wie soziale Kontakte, Bestätigung und Anerkennung. So wie Ältere die Selbständigkeit und Unabhängigkeit für sich bewahren wollen (Matthäi 2005), so wünschen sie dies auch für ihre Kinder. Die zweite Migrantengeneration befindet sich mit ihren Einstellungen dabei zwischen der ersten Migrantengeneration einerseits und den Deutschen andererseits: Nur in diesem Item sind Veränderungen zu konstatieren, die vermutlich als Akkulturationsprozesse zu deuten sind. Insgesamt bleibt allerdings die Versorgung der Älteren bei türkischen Migranten viel stärker als bei Deutschen Familiensache (Zimmermann 2012). So unterstreichen die Daten, dass Transmissionsprozesse stärker sind als Akkulturationsprozesse zwischen den Generationen, oder letztere gemeinsam im Konvoi durchlaufen werden (Nauck 2000). ${ }^{3}$

3 Weitergehende Analysen, die für die erste Generation türkischer Migranten die Dauer des Aufenthaltes in Deutschland bzw. der Sprachkenntnisse als Akkulturationsindikatoren berücksichtigten, zeigten übrigens keinen Effekt der Aufenthaltsdauer und einen geringen negativen Effekt guter Deutschkenntnisse, allerdings nur für das Item „Eltern aufnehmen“ $(p<0,10)$. 
Dabei stellt sich natürlich die Frage, ob das tatsächliche Verhalten zur Pflege mit den Einstellungen korrespondiert. Einstellungen sind ein eher abstraktes Maß zum Messen von Verhalten, was nicht zum entsprechenden Verhalten führen muss (Ajzen 1985; Frey et al. 2001). Faktisch ist bisher unklar, in welchem Umfang in türkischen Familien zusätzlich außerfamiliäre Hilfe in Anspruch genommen wird. Regionale Daten zeigen, dass türkische Migranten seltener als pflegebedürftig anerkannt werden und häufiger eine geringere Pflegestufe erhalten. Geldleistungen werden häufiger beantragt als Sachleistungen und stationäre Pflege ist sehr selten (Okken et al. 2008). Verschiedene qualitative Studien unterstreichen, dass Pflege als häusliche und familiäre Angelegenheit betrachtet wird, vor dem Hintergrund einer starken Familienorientierung und -bindung. Größte Sorge vieler Älterer ist zugleich die Abhängigkeit von Kindern. Deren (geschlechtsspezifische) Belastungen, wie Arbeits- und Familienverpflichtungen, aber auch für eine intensivere Unterstützung und ungeeignete Wohnbedingungen werden perzipiert und stellen Hinderungsgründe für eine auch von Kindern durchaus gewünschte familiäre Pflege dar (Zimmermann 2012). In der Folge finden sich Überforderung der Familien und Verdrängung, die einer eigentlich notwendigen Vorbereitung entgegenstehen. So wird vielfach, um sich nicht gegenseitig zu enttäuschen, die Frage der Pflegebedürftigkeit gar nicht thematisiert (Paß 2006).

Die starke familiale Solidarität, die in diesen Daten gezeigt werden konnte, ist eine wichtige Ressource, die es durch ergänzende Hilfen zu erhalten und unterstützen gilt. Trotz einer inzwischen langen Diskussion über die Erfordernisse einer kultursensiblen Pflege (Zeman 2012) bedarf es weiterhin sowohl umfassender Informationen über Ansprüche gegenüber Alten- und Pflegehilfe an ältere Migranten und ihre Familien, als auch entsprechende Angebote im Rahmen eines Diversity Management, das die spezifischen Bedarfe von sprachlich-kultursensiblen Hilfsangeboten und Pflege berücksichtigt, v.a. im Fall von Demenzerkrankungen (Kaiser 2009). Von zentraler Bedeutung sind familienund frauenunterstützende Maßnahmen. Es geht um das Zusammenspiel von familiärer Solidarität und formeller Unterstützung. Mit wachsenden Anforderungen an sowohl die innerfamiliäre als auch die staatlichen Unterstützungsquellen ist für die Zukunft zu rechnen.

\section{Literatur}

Aboderin, I. (2004). Modernisation and ageing theory revisited: Current explanation of recent developing world and historical western shifts in material family support of older people. Aging and Society, 24, S. $29-50$.

Ajzen, I. (1985). From intentions to actions: A theory of planned behavior. In: Kuhl, J. \& Beckmann J. (Hrsg.), Action control: From cognition to behavior. Heidelberg: Springer Verlag, S. 11-39.

Alba, R. \& Nee, V. (1997). Rethinking assimilation theory for a new era of immigration. International Migration Review, 31, S. 826-874.

Angel, J. L., Angel, R. J., McClellan, J. L. \& Markides, K. S. (1996). Nativity, declining health, and preferences in living arrangements among elderly Mexican Americans: Implications for long-term care. The Gerontologist, 36, S. 464-473.

Arends-Toth, J. \& Van de Vijver, F. J. R. (2008). Family relationships among immigrants and majority members in the Netherlands: The role of acculturation. Applied Psychology: An International Review 57, S. 466-487. 
Ataca, B. (2006). Turkey. In: Georgas, J. E. A. (Hrsg.), Families across cultures. A 30-nation psychological study. Cambridge: University Press: S. 467-474.

Baykara-Krumme, H. (2007). Gar nicht so anders: Eine vergleichende Analyse der Generationenbeziehungen bei Migranten und Einheimischen in der zweiten Lebenshälfte. Berlin: Wissenschaftszentrum Berlin für Sozialforschung (Discussions Paper Nr. SP IV 2007-604).

Baykara-Krumme, H. \& Hoff, A. (2006). Die Lebenssituation älterer Ausländerinnen und Ausländer in Deutschland. In: Tesch-Römer, C., Engstler, H. \& Wurm, S. (Hrsg.), Altwerden in Deutschland. Sozialer Wandel und individuelle Entwicklung in der zweiten Lebenshälfte. Wiesbaden: VS Verlag für Sozialwissenschaften, S. 447-517.

Baykara-Krumme, H.,Klaus, D. \& Steinbach, A. (2011). Generationenbeziehungen in Deutschland. Ein Vergleich der Beziehungsqualität in einheimischen deutschen Familien, Familien mit türkischem Migrationshintergrund und Aussiedlerfamilien. In: Brüderl, J., Castiglioni, L. \& Schumann, N. (Hrsg.), Partnerschaft, Fertilität und intergenerationale Beziehungen. Würzburg: Ergon, S. 259286.

Berry, J. W. (1997). Immigration, acculturation, and adaptation. Applied Psychology. An International Review 46, S. 5-34.

Bundesministerium für Familie, Senioren, Frauen und Jugend (Hrsg.) (2000). Sechster Familienbericht. Familien ausländischer Herkunft in Deutschland. Leistung - Belastung - Herausforderung. Berlin.

Bundesministerium für Familie, Senioren, Frauen und Jugend (Hrsg.) (2005). Fünfter Bericht zur Lage der älteren Generation in der Bundesrepublik Deutschland: Potenziale des Alters in Wirtschaft und Gesellschaft. Berlin: Bundesministerium für Familie, Senioren, Frauen und Jugend.

Bundesministerium für Familie, Senioren, Frauen und Jugend (Hrsg.) (2010). Ehe, Familie, Werte Migrantinnen und Migranten in Deutschland. Berlin: Bundesministerium für Familie, Senioren, Frauen und Jugend.

Bundesamt für Migration und Flüchtlinge (Hrsg.) (2010). Migrationsbericht des Bundesamts für Migration und Flüchtlinge im Auftrag der Bundesregierung. Berlin: Bundesministerium des Inneren.

Burr, J. A. \& Mutchler, J. E. (1999). Race and ethnic variation in norms of filial responsibility among older persons. Journal of Marriage and the Family 61, S. 647-687.

Cicirelli, V.G. (1990). Family support in relation to health problems of the elderly. In: Brubaker, T. H. (Hrsg.), Family relationship in later life. Newbury Park: Sage Publications, S. 212-222.

De Jong-Gierveld, J. \& de Kampbuis, F. (1985). The development of a Rasch-type loneliness scale. Applied Psychological Measurement, 9, 3, S. 289-299.

De Jong-Gierveld, J. \& van Tilburg, T. (2006): A 6-item scale for overall, emotional, and social loneliness: Confirmatory tests on survey data. Research on Aging, 28, 5, S. 582-598.

De Valk, H.A. \& Schans, D. (2008). "They ought to do this for their parents": Perceptions of filial obligations among immigrant and Dutch older people. Ageing and Society, 28, S. 49-66.

Dietzel-Papakyriakou, M. (1993). Altern in der Migration. Die Arbeitsmigranten vor dem Dilemma: zurückkehren oder bleiben? Stuttgart: Ferdinand Enke Verlag.

Dykstra, P. A. (2009). Older adult loneliness: Myths and realities. European Journal of Aging 6, S. 91100.

Ette, A., Hullen, G., Leven, I. \& Ruckdeschel, K. (2007). Generations and Gender Survey. Dokumentation der Befragung von türkischen Migranten in Deutschland. Wiesbaden: Bundesinstitut für Bevölkerungsforschung.

Frey, D., Stahlberg, D. \& Gollwitzer P. M. (2001). Einstellung und Verhalten: Die Theorie des überlegten Handelns und die Theorie des geplanten Verhaltens. In: Frey, D. \& Irle, M. (Hrsg.), Kognitive Theorien der Sozialpsychologie. Band 1. Bern: Verlag Hans Huber, S. 361-398.

Glick, J. E. (2010). Connecting complex processes: A decade of research on immigrant families. Journal of Marriage and Family 72, 3, S. 498-515.

Glick, J. E. \& Van Hook, J. (2002). Parents' coresidence with adult children: Can immigration explain racial and ethnic variation? Journal of Marriage and Family, 64 S. 240-253. 
Idema, H. \& Phalet, K. (2007). Transmission of gender-role values in Turkish-German migrant families: The role of gender, intergenerational and intercultural relations. Zeitschrift für Familienforschung/Journal of Family Research 19, 1, S. 71-105.

Inglehart, R. \& Baker, W. E. (2000). Modernization, cultural change and the persistence of traditional values. American Sociological Review, 65, 1, S. 19-51.

Jylhä, M. \& Saarenheimo, M. (2010): Loneliness and aging: Comparative perspectives. In: Dannefer, D. \& Phillipson, C. (Hrsg.), The Sage handbook of social gerontology. London: Sage, S. 317-328.

Kağıtçıaşı, Ç. (1996). Family and human development across cultures: A view from the other side. Mahwah, New Jersey: Lawrence Erlbaum Associates.

Kağıtçıbaşı, Ç. (2006). Theoretical perspectives on family change. In: Georgas, J. E. A. (Hrsg.), Families across cultures. A 30-nation psychological study. Cambridge: University Press, S. 72-89.

Kaiser, C. (2009). Ältere Migranten und Demenz. Versorgungssituation, Handlungsbedarf und erste Modellprojekte. Saarbrücken: VDM Verlag.

Kao, H. S. \& Travis, S. (2005). Effects of acculturation and social exchange on the expectations of filial piety among Hispanic/Latino parents of adult children. Nursing/Health Sciences, 7, S. 226-234.

Katz, R. (2009). Intergenerational family relations and life satisfaction among three elderly population groups in transition in the Israeli multi-cultural society. Journal of Cross-Cultural Gerontology, 24, S. 77-91.

Kofman, E. (2004). Family-related migration: A critical review of European studies. Journal of Ethnic and Migration Studies 20, 3, S. 243-262.

Kreyenfeld, M., Hornung, A., Kubisch, K. \& Jaschinski, I. (2010). Fertility and union histories from German GGS data: Some critical reflections. Rostock: MPIDR (MPIDR Working Paper 2010-023).

Lye, D. N. (1996). Adult child-parent relationships. Annual Review of Sociology, 22, S. 79-102.

Laidlaw, K., Wang, D., Coelho, C. H. \& Power, M. (2010). Attitudes to ageing and expectations for filial piety across Chinese and British cultures: A pilot exploratory evaluation. Aging \& Mental Health, 14,3, S. 283-292.

Liefbroer, A. C. \& Mulder, C. H. (2006). Family obligations. In: Dykstra, P.A., Kalmijn, M. T., Knijn, C. M., Komter, A. E., Liefbroer, A. C. \& Mulder C.H. (Hrsg.). Family solidarity in the Netherlands. Amsterdam: Dutch University Press, S. 123-146.

Markus, H. R. \& Kitayama, S. (1991). Culture and the self: Implications for cognition. emotion, and motivation. Psychological Review 98, S. 224-253.

Matthäi, I. (2005). Die vergessenen Frauen aus der Zuwanderergeneration. Zur Lebenssituation von alleinstehenden Migrantinnen im Alter. Wiesbaden: VS Verlag für Sozialwissenschaften.

Milewski, N. (2009). Fertility of immigrants. A two-generational approach in Germany. Heidelberg: Springer.

Naderi, R., Dorbritz, J. \& Ruckdeschel, K. (2009). Einleitung. Der Generations and Gender Survey in Deutschland: Zielsetzung, Verortung, Einschränkungen und Potenziale. Zeitschrift für Bevölkerungswissenschaft $34,1-2$, S. 5-30.

Nauck, B. (2000). Eltern-Kind-Beziehungen in Migrantenfamilien - ein Vergleich

zwischen griechischen, italienischen, türkischen und vietnamesischen Familien in Deutschland. In: Sachverständigenkommission, 6. Familienbericht (Hrsg.), Empirische Beiträge zur Familienentwicklung und Akkulturation. Materialien zum 6. Familienbericht, Band 1. Opladen: Leske \& Budrich, S. 347-392.

Nauck, B. (2001). Solidarpotenziale von Migrantenfamilien. Bonn: Friedrich-Ebert-Stiftung (Expertise).

Nauck, B. (2002). Dreißig Jahre Migrantenfamilien in der Bundesrepublik. Familiärer Wandel zwischen Situationsanpassung, Akkulturation, Segregation und Remigration. In: Nave-Herz, R. (Hrsg.), Kontinuität und Wandel der Familie in Deutschland. Eine zeitgeschichtliche Analyse. Stuttgart: Lucius und Lucius, S. 315-339.

Nauck, B. \& Suckow, J. (2006). Intergenerational relationships in cross-cultural comparison: How social networks frame intergenerational relations between mothers and grandmothers in Japan, Korea, China, Indonesia, Israel, Germany, and Turkey. Journal of Family Issues, 27, S. 1159-1185. 
Okken, P.-K., Spallek, J. \& Razum, O. (2008). Pflege türkischer Migranten. In: Bauer, U. \& Büscher, A. (Hrsg.). Soziale Ungleichheit und Pflege: Beiträge sozialwissenschaftlich orientierter Pflegeforschung. Wiesbaden: VS Verlag für Sozialwissenschaften, S. 396-422.

Olbermann, E. (2003). Soziale Netzwerke, Alter und Migration. Theoretische und empirischen Exploration zur sozialen Unterstützung älterer Migranten. Dortmund: Universität Dortmund (Dissertation).

Park, P. E. (1964). Race and culture. New York: The Free Press of Clencoe.

$\mathrm{Paß}$, R. (2006). Alter(n)svorstellungen älterer Migrantinnen. Eine explorative Studie über deren biografische Lebensentwürfe. Osnabrück: Verlag Dr. Kovač.

Portes, A. \& Zhou, M. (2005). The new second generation: Segmented assimilation and its variants. In: Suárez-Orozco, M. M., Suárez-Orozco, C. \& Baolian Qin, D. (Hrg.), The new immigration. New York: Brunner-Routledge, S. 85-103.

Portes, A. \& Rumbaut, R.G. (2001). Legacies. The story of the immigrant second generation. Berkeley u.a.: University of California Press.

Prahl, H. W.\& Schroeter, K. R. (1996). Soziologie des Alterns. Eine Einführung. Paderborn u.a.: Schöningh.

Rossi, A. S. \& Rossi, P. H. (1990). Of human bonding - Parent-child relations across the life-course. New York: Aldine de Gruyter.

Robert-Koch-Institut (Hrsg.) (2008). Migration und Gesundheit: Schwerpunktbericht der Gesundheitsberichterstattung des Bundes. Berlin: Robert-Koch-Institut (in Zusammenarbeit mit dem Statistischen Bundesamt).

Sauer, L., Ruckdeschel, K. \& Naderi, R. (2012). Reliability of retrospective event histories within the German Generations and Gender Survey. Wiesbaden: Bundesinstitut für Bevölkerungsforschung (BiB Working Paper 1/2012).

Schrader, A., Nickles, B.\& Griese, H. (1979). Die zweite Generation. Sozialisation und Akkulturation ausländischer Kinder in der Bundesrepublik. Königstein: Athenäum Verlag.

Statistisches Bundesamt (2011). Bevölkerung und Erwerbstätigkeit. Bevölkerung mit Migrationshintergrund - Ergebnisse des Mikrozensus 2010. Wiesbaden: Statistisches Bundesamt.

Steinbach, A. (2004). Solidarpotenziale in Migrantenfamilien. In: Krüger-Potratz, M. (Hrsg): Familien in der Einwanderungsgesellschaft. Beiträge der Akademie für Migration und Integration. Heft 8, Göttingen: V\&R unipress, S. 39-48.

Szydlik, M. (2000). Lebenslage Solidarität? Generationenbeziehungen zwischen erwachsenen Kindern und Eltern. Opladen: Leske + Budrich.

Tardif-Williams, C. Y. \& Fisher, L. (2009). Clarifying the link between acculturation experiences and parent-child relationships among families in cultural transition: The promise of contemporary critiques of acculturation psychology. International Journal of Intercultural Relations 33, 2, S. 150-161.

Tucci, I. \& Yildiz, S. (2012). Das Alterseinkommen von Migrantinnen und Migranten: zur Erklärungskraft von Bildungs- und Erwerbsbiografien. In: Baykara-Krumme, H., Motel-Klingebiel, A. \& Schimany, P. (Hrsg.), Viele Welten des Alterns? Ältere Migranten im alternden Deutschland. Wiesbaden, VS Verlag für Sozialwissenschaften, S. 101-126.

UNESCO. International Standard Classification of Education ISCED. www.circa.europa.eu/irc/dsis/ employment/info/data/eu_lfs/lfs_main/Related_documents/ISCED_EN.htm (Stand: 2012-07-15).

Vogel, C. (2012). Generationenbeziehungen der (Spät-)Aussiedler. Forschungsstand und exemplarische Befunde zu Einstellungen in Bezug auf familiale Unterstützungsleistungen. In: Baykara-Krumme, H., Motel-Klingebiel, A. \& Schimany, P. (Hrsg.), Viele Welten des Alterns. Ältere Migranten im alternden Deutschland. Wiesbaden: Springer VS, S. 289-313.

Zeman, P. (2009). Ältere Menschen mit Migrationshintergrund. Soziale Arbeit, 11-12, S. 435-445.

Zeman, P. (2012). Ältere Migrantinnen und Migranten in der Altenhilfe und kommunalen Altenpolitik. In: Baykara-Krumme, H., Motel-Klingebiel, A. \& Schimany, P. (Hrsg.), Viele Welten des Alterns. Ältere Migranten im alternden Deutschland. Wiesbaden: Springer VS, S. 449-465.

Zhou, M. (1997). Growing up American: The challenge confronting immigrant children and children of immigrants. Annual Review of Sociology 23, S. 63-95. 
Zimmermann, H.-P. (2012). Altersbilder von türkischen Migrantinnen und Migranten in Deutschland im Vergleich. Islamische Grundsätze - alltägliche Sichtweisen. In: Baykara-Krumme, H., MotelKlingebiel, A. \& Schimany, P. (Hrsg.), Viele Welten des Alterns? Ältere Migranten im alternden Deutschland. Wiesbaden: Springer VS, S. 315-337.

Eingereicht am/Submitted on: 23.07.2012

Angenommen am/Accepted on: 29.01.2013

Anschriften der Autorinnen/Addresses of the authors:

Marie Carnein, M. A. (Korrespondierende Autorin/Corresponding author)

Universität Rostock

Institut für Allgemeine Pädagogik und Sozialpädagogik

August-Bebel-Straße 28

18055 Rostock

Deutschland/Germany

Dr. Helen Baykara-Krumme

Technische Universität Chemnitz

Institut für Soziologie

Thüringer Weg 9

09126 Chemnitz

Deutschland/Germany

E-Mail: marie.carnein@uni-rostock.de helen.baykara@soziologie.tu-chemnitz.de 